\title{
Treatment Strategy for Patients with Acute Myocardial Infarction
}

\author{
Kazuyuki Shirai and Keijiro Saku \\ Key words: thrombolytic therapy, percutaneous coronary intervention (PCI), intravenous coronary thromboly- \\ sis (IVCT), intra-coronary thrombolysis (ICT)
}

(DOI: 10.2169/internalmedicine.45.0157)

Reperfusion therapy has been proven to reduce mortality and to improve cardiac function in patients with acute myocardial infarction (AMI). Reperfusion therapy encompasses both thrombolytic therapy and percutaneous coronary intervention (PCI).

\section{Thrombolytic Therapy}

Intravenous coronary thrombolysis (IVCT) for AMI is a simple procedure and can be performed in most institutions. In America and Europe, many clinical trials have been performed to test the safety and efficacy of thrombolytic therapy. Research by the Fibrinolytic Therapy Trialists analyzed 9 randomized clinical trials of more than 1,000 patients and reported that thrombolytic therapy significantly reduced 5week mortality compared to a placebo in 58,600 patients with possible AMI (1). This analysis showed that thrombolytic therapy was effective, especially in patients with ST elevation or bundle branch block in electrocardiography, and was more effective when the thrombolytic therapy was performed soon after the onset of myocardial infarction.

For optimal results, reperfusion therapy should be performed as soon as possible after the onset of AMI. Prehospital thrombolysis is defined as the administration of a thrombolytic agent before a patient arrives at the hospital. In the early era, pre-hospital thrombolysis was thought to be associated with an increase in the rate of sudden worsening due to reperfusion arrhythmia, reperfusion injury or bleeding complications during transport. However, a meta-analysis of many randomized clinical trials of pre-hospital versus inhospital thrombolysis for 6,434 AMI patients showed that pre-hospital thrombolysis shortened the time to thrombolytic therapy and significantly reduced hospital mortality when performed safely and appropriately (2). A low incidence of successful reperfusion with TIMI 3 flow and bleeding complications are the main issues with thrombolytic therapy.
Intra-coronary thrombolysis (ICT) required less thrombolytic agent than IVCT and was thought to be useful because it was associated with a higher incidence of successful reperfusion and fewer bleeding complications compared to IVCT. However, ICT can be performed only in limited institutions and the time to treatment is longer than with IVCT. Percutaneous coronary intervention (PCI) is associated with a higher incidence of successful reperfusion and fewer bleeding complications compared to ICT, and the frequency of ICT has declined.

\section{Percutaneous Coronary Intervention (PCI)}

PCI has been demonstrated to be superior to thrombolytic therapy with regard to the restoration of TIMI 3 flow and is associated with lower rates of recurrent ischemia, reinfarction, stroke, and death, although the time from the onset of myocardial infarction can be considerably long because hospital emergency departments are not always equipped to perform coronary angiography. Coronary stent implantation improved the outcome of patients with PCI, and has also been proven to be effective in patients with AMI in the STENT PAMI trial (3).

One of the issues regarding PCI for patients with MI is no reflow, which is associated with distal embolism caused by a large amount of intracoronary thrombus or crushed plaque. Distal protection devices have been developed to avoid distal embolism and are expected to improve the patient outcome. However, in the EMERALD trial (4), a distal protection device was tested in patients with AMI within 6 hours from onset, but was not shown to be effective.

Many trials have been performed to compare primary PCI with thrombolytic therapy for AMI. A review of 23 randomized trials that compared PCI to thrombolytic therapy in patients with ST elevation in MI showed that primary PCI was associated with low incidences of short- and long-term 
death, and reinfarction or cerebrovascular accident compared to thrombolytic therapy (5).

This study appeased on the issue (6) compared the outcomes of reperfusion therapy with IVCT and ICT versus primary PCI. The IVCT group was associated with greater TIMI 3 flow at the initial angiogram and with a lower incidence of short-term death. One of the most important factors to affect these results is thought to be the time from the onset of myocardial infarction to reperfusion therapy. The average time from symptom onset to reperfusion therapy in the IVCT group (227 minutes) was more than 4 hours shorter than that in the primary PCI group (479 minutes). The primary PCI group may have had more patients with AMI more than 12 hours from the onset, in whom the efficacy of reperfusion therapy is not clear. In the CAPTIM trial (7), patients who were randomized $<2$ hours after symptom onset had a trend toward lower 30-day mortality with pre-hospital thrombolysis compared to those randomized to primary PCI. In addition, among patients who were randomized in the first 2 hours, cardiogenic shock was less frequent with thrombolytic therapy than with primary PCI.

This seemed to affect the finding in this study that more than $70 \%$ of the patients in the IVCT group underwent PCI after thrombolytic therapy. In the REACT trial (8), rescue PCI after failed thrombolytic therapy significantly reduced the incidence of composite events of death, reinfarction, stroke, or severe heart failure.

\section{Combination Therapy with Thrombolysis and PCI}

According to clinical trials in the 1980's, such as the
TIMI 2 and PAMI trials, PCI in patients with AMI after thrombolysis resulted in increased rates of death, reinfarction, emergency bypass and blood transfusion, and thus this procedure was considered to be an undesirable method of therapy. However, with the introduction of the stent and advances in technology, a combination therapy with thrombolysis and PCI has been reconsidered. Gasior et al (9) compared primary PCI and PCI after initial thrombolysis in patients with AMI. In this study, combination therapy with thrombolysis and PCI was associated with a high incidence of TIMI 3 flow after the procedure without an increase in complications.

\section{Conclusions}

The prognosis of patients with myocardial infarction has dramatically improved due to the development of reperfusion therapy. With the development of new medicine and medical devices, reperfusion therapy is expected to provide a better method of treatment in the future. It is clear that the most important factor in reperfusion therapy is the time from onset to the time of the procedure and achieving TIMI 3 flow. Therefore, combination therapy with pre-hospital thrombolysis and PCI is thought to be the best procedure of choice at this time. However, there are various situations in individual institutions with respect to time and place, transport of the patient, and the medical facilities available. Especially in Japan, hospital available PCI is not rare, and transport time of the victim of out-of-hospital ACS to the hospital emergency department is generally less than one hour. Thus, the medical procedure to be performed should be determined according to the situation.

\section{References}

1. Group FC. Indications for fibrinolytic therapy in suspected acute myocardial infarction: collaborative overview of early mortality and major morbidity results from all randomised trials of more than 1000 patients. Fibrinolytic Therapy Trialists' (FTT) Collaborative Group. Lancet 343: 311-322, 1994.

2. Cannon CP, Gibson CM, Lambrew CT, et al. Relationship of symptom-onset-to-balloon time and door-to-balloon time with mortality in patients undergoing angioplasty for acute myocardial infarction. JAMA 283: 2941-2947, 2000.

3. Grines CL, Cox DA, Stone GW, et al. Coronary angioplasty with or without stent implantation for acute myocardial infarction. Stent Primary Angioplasty in Myocardial Infarction Study Group. N Engl J Med 341: 1949-1956, 1999.

4. Stone GW. Primary angioplasty in acute myocardial infarction with distal protection of the microcirculation: Principal results from the prospective, randomized EMERALD Trial. JACC 43: 285A, 2004.

5. Keeley EC, Boura JA, Grines CL. Primary angioplasty versus in- travenous thrombolytic therapy for acute myocardial infarction: a quantitative review of 23 randomised trials. Lancet 361: 13-20, 2003.

6. Kobayashi H, Takazawa K, Matsumoto C, et al. The role of intravenous coronary thrombolysis for patients with acute myocardial infarction in different treatment strategies. Intern Med 45: 709714, 2006.

7. Steg PG, Bonnefoy E, Chabaud S, et al. Impact of time to treatment on mortality after prehospital fibrinolysis or primary angioplasty: data from the CAPTIM randomized clinical trial. Circulation 108: 2851-2856, 2003.

8. Gershlick AH, Stephens-Lloyd A, Hughes S, et al. Rescue angioplasty after failed thrombolytic therapy for acute myocardial infarction. N Engl J Med 353: 2758-2768, 2005.

9. Gasior M, Gierlotka M, Lekston A, et al. Relation between time of pain and long-term outcome in patients with acute myocardial infarction transferred to primary angioplasty or angioplasty facilitated with thrombolysis. JACC 43: 264A, 2004.

(C) 2006 The Japanese Society of Internal Medicine http://www.naika.or.jp/imindex.html 